\section{GIVE A GAME CHANGER THIS CHRISTMAS}

This year's festive offering from Philips is a dual AirFloss and Sonicare HealthyWhite power pack which offers patients and their family and friends the potential of both cleaner and whiter teeth in combination. The pack is accompanied by a 'Gifts They'll Love' point of sale tag to highlight its potential as the perfect Christmas present.

AirFloss - which dental professionals have called a 'game changer' because it has such intrinsic clinical benefits - shoots a jet of air laden with water (or mouthwash) to clean deep between the teeth making it much easier to remove plaque bacteria and get between every tooth in a minute.

The Sonicare HealthyWhite features a dedicated whitening mode which makes it possible to remove extrinsic stains, resulting in a brighter, whiter smile. This celebrated sonic toothbrush has a special extended 30 seconds setting which is so effective users can lighten their teeth by two shades in just two weeks and ensure that every quadrant of the mouth is expertly cleaned in the process.

The combo pack has a high street value of $£ 200$ and is available from the beginning November for the price of $£ 99$, making it the most desirable Christmas double act since Morecambe and Wise. To obtain the dual pack call 0800 0567222 or speak to your local representative.

\title{
FREE TRAINING IN PAIN-FREE ANAESTHESIA
}

General Medical have commissioned a Training DVD for Quicksleeper, the computer controlled local anaesthetic system that delivers profound anaesthesia quickly, easily and painlessly.

Available free of charge, it describes the theory behind the technique and demonstrates the Quicksleeper in use for a

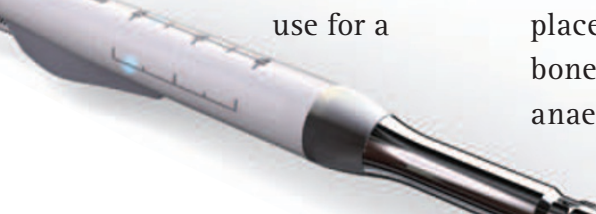
bone in between teeth. This delivers a profound naesthesia almost instantaneously.

To request your Free Training DVD call 01380 734990 or visit www.generalmedical.co.uk.

\section{A SHARP FOCUS ON SOFTWARE ESSENTIALS}

The defining features of Sirona's CEREC SW 4.0 software are a sharp focus on essentials, attractive and modern design, plus the streamlined production of clinically and aesthetically perfect dental restorations. A key highlight is the intuitive user interface, which has been redesigned using the latest software development tools. All the settings and processing steps are visualised with the aid of self-explanatory icons and photorealistic images. As a result, newcomers will find it easy to get to grips with the CEREC system - and experienced users will be able to achieve even more convincing results than in the past.

The CEREC system enables dentists to create all-ceramic restorations directly 'in-house'. The New CEREC SW 4.0 system guides the user step by step through the entire production procedure. The software functions and options adapt themselves dynamically as the work proceeds. The user interface remains clear and uncluttered, and only those items that are relevant are displayed at any given time.

To cater for patients with multiple clinical indications the new software allows dentists to work in parallel on several different restorations during a single treatment session. As a result they are ideally placed to offer their patients a complete portfolio of aesthetic and functionally effective therapy solutions.

Appealing to experienced and new users alike, a software upgrade to CEREC SW 4.0 is available free of charge to CEREC Club members.

For further information contact Sirona Dental Systems on 08450715040 or email info@sironadental.co.uk.

\section{THE IDEAL}

\section{CEMENTATION CHOICE}

GC Fuji TEMP LT counts on the well-known safety offered by glass-ionomer materials. It has all you need in temporary cement: pleasant handling, no runny consistency with low film thickness, easy to remove excess, reliable retention, long term security, no negative influence on final adhesive cementation, secure retrievability, safe removal of the restoration whenever needed, and easy clean up.

These advantages combined with the safety offered by glass ionomers make it the ideal choice for long-term temporary cementation of all types of all-ceramic, resin, acrylic and metal-based crowns and bridges, including try-on cementation of long-span prosthetic appliances; and especially adapted to assure sufficient retention and retrievability of crowns and bridges cemented on implant abutments.

GC Fuji TEMP LT comes in one single shade (Universal) and is presented in paste pack cartridges to be used with the practical Paste Pack Dispenser from GC. Removal of cemented prosthetic appliances is better performed with the use of GC Pliers for better control of applied forces.

Contact GC UK on 01908 218999 orinfo@uk.gceurope.com. 